Abanico Veterinario. Enero-Diciembre 2021; 11:1-16. http://dx.doi.org/10.21929/abavet2021.13 Artículo Original. Recibido: 02/12/2020. Aceptado: 20/03/2021. Publicado: 01/04/2021. Clave: e2020-95.

\title{
Interacción genotipo por ambiente en camarón blanco asociada a Síndrome de Mancha Blanca
}

\author{
Genotype-by-environment interaction in white shrimp associated with White Spot \\ Syndrome
}

\section{Cala-Moreno Nelson ${ }^{* 11 D}$, Campos-Montes Gabriel ${ }^{21 D}$, Caballero-Zamora Alejandra $^{31 D}$, Berruecos-Villalobos José ${ }^{11 D}$, Castillo-Juárez Hector ${ }^{31 D \S}$}

1Departamento de Genética y Bioestadística, Facultad de Medicina Veterinaria y Zootecnia, Universidad Nacional Autónoma de México. México. ${ }^{2}$ Departamento El Hombre y su Ambiente, Universidad Autónoma Metropolitana, Xochimilco, México. ${ }^{3}$ Departamento de Producción Agrícola y Animal, Universidad Autónoma Metropolitana, Xochimilco, México. §Fallecido, marzo 18, 2019. *Autor para la correspondencia: Nelson Cala Moreno. Departamento de Genética y Bioestadística, Facultad de Medicina Veterinaria y Zootecnia, Universidad Nacional Autónoma de México, Avenida Universidad \#3000, CP 04510, México. Email: necamo1980@gmail.com, nonino@prodigy.net.mx, gabocamo@gmail.com, alejandra.caballero.zamora@gmail.com

\section{RESUMEN}

Este estudio tuvo como objetivo estimar la interacción genotipo por ambiente para peso corporal (PC) y supervivencia a cosecha (SC), en presencia y ausencia de Síndrome de Mancha Blanca (SMB) en dos líneas genéticas de Penaeus vannamei (Crecimiento -CRE- y resistencia a SMB -RES-). La heredabilidad para PC en la línea CRE fue $0.05 \pm 0.16$ en presencia de SMB y $0.35 \pm 0.15$ en ausencia, mientras que para la línea RES fueron $0.26 \pm 0.07$ y $0.49 \pm 0.08$ en presencia y ausencia de SMB, respectivamente. Las correlaciones genéticas para PC entre ambientes fueron $-0.17 \pm 0.60$ para CRE y $0.89 \pm 0.09$ para RES. La heredabilidad para SC en CRE fue 0.01 en ambos ambientes y la correlación genética no fue estimable, mientras que, para RES, las heredabilidades fueron $0.06 \pm 0.04$ y $0.11 \pm 0.06$ en ausencia de y presencia de SMB, respectivamente, adicionalmente, la correlación genética no fue significativa. Aunque el modelo lineal sugiere una interacción genotipo por ambiente, los estimados proponen independencia de la misma característica entre ambientes y, las correlaciones entre características para la línea de resistencia proponen seleccionar de manera independiente las características cuando SMB esté presente.

Palabras clave: Penaeus vannamei, heredabilidad, correlación genética aditiva, peso corporal, supervivencia.

\begin{abstract}
This study aimed to estimate genotype-by-environment interaction for body weight (BW) and survival in presence and absence of White Spot Disease (WSD) in two genetic lines of Penaeus vannamei (Growth GRO- and resistance to WSD -RES). Heritability for BW in the GRO line was $0.05 \pm 0.16$ in the presence of WSD and $0.35 \pm 0.15$ in the absence, while for the RES line they were $0.26 \pm 0.07$ and $0.49 \pm 0.08$ in the presence and absence of WSD, respectively. Genetic correlations for BW between environments were $-0.17 \pm 0.60$ for GRO and $0.89 \pm 0.09$ for RES. Heritability for survival in GRO was 0.01 in both environments and the genetic correlation was not estimable, whereas, for RES, the heritabilities were $0.06 \pm 0.04$ and $0.11 \pm 0.06$ in the absence and presence of WSD, respectively, additionally, the genetic correlation was not significant. Although the linear model suggests a genotype-by-environment interaction, the estimates propose independence of the same trait between environments, and the correlations between traits in the resistance line propose to independently select traits when WSD is present.
\end{abstract}

Keywords: Penaeus vannamei, heritability, additive genetic correlation, body weight, survival. 


\section{INTRODUCCIÓN}

La producción mundial de camarón blanco del Pacífico (Penaeus vannamei) se ha basado en la producción de líneas genéticas que han sido seleccionadas para crecimiento y supervivencia general (Campos-Montes et al., 2009; Caballero-Zamora et al., 2015; Yuan et al., 2018). Al mismo tiempo, las unidades de producción han sido afectadas por varias enfermedades con altas tasas de morbilidad y mortalidad (Trang et al., 2019); entre ellas, el Síndrome de Mancha Blanca (SMB) (Hernández-Llamas et al., 2016).

El control de SMB ha sido una meta difícil y se ha optado por añadir criterios de selección relacionados a la resistencia de esta enfermedad al objetivo de selección de Programas de Mejoramiento Genético (PMG) en peneidos (Ødegård et al., 2011; Huang et al., 2012; Klinger y Naylor, 2012; Caballero-Zamora et al., 2015).En esta idea, es importante tener estimadores adecuados de heredabilidad $\left(h^{2}\right)$ y correlación genética ( $\mathrm{rG}$ ) para la formulación de estrategias de selección. Estos parámetros genéticos, estimados en condiciones de brote natural, pueden brindar información importante para ser considerada en los PMG. Algunos autores han estimado la heredabilidad para peso corporal en presencia del SMB entre 0.09, usando información de brote y 0.21 a partir de un desafío controlado (Gitterle et al., 2005b; Caballero-Zamora et al., 2015).

En cuanto a la heredabilidad para supervivencia en presencia del SMB, ha sido estimada entre 0.01 y 0.21 en estudios de desafío controlado a partir de diferentes modelos estadísticos y protocolos de infección (Gitterle et al., 2005b; Gitterle et al., 2006a; Gitterle et al., 2006b), y como 0.06 en condiciones de brote natural del SMB (Caballero-Zamora et al., 2015). En cambio, la estimación de estos parámetros para la misma característica en ambientes diferentes puede interpretarse como una interacción de genotipo por ambiente (IGA). Dicha IGA, puede modificar la estimación de $h^{2}$ y rG entre criterios de selección, provocando respuestas a la selección poco precisas y alteraciones en el ordenamiento de los candidatos a reproductores (Sae-Lim et al., 2016).

En P. vannamei, estudios previos han buscado IGA para peso corporal a la cosecha (PC) entre localidades o densidades de siembra en condiciones comerciales, sin hallar evidencia (Ibarra y Famula, 2008; Campos-Montes et al., 2009). No obstante, CaballeroZamora et al. (2015) observaron efectos de IGA para peso corporal a las 19 semanas de edad entre poblaciones que crecieron en presencia 0 en ausencia del SMB en condiciones comerciales. En cuanto a IGA para supervivencia general a la cosecha (SC), ningún estudio ha reportado efectos de IGA.

En cuanto a la ra para peso y supervivencia en camarón, algunos estudios han estimado rG entre PC y SC en ausencia de alguna enfermedad entre -0.49 y 0.56 (Campos-Montes et al., 2013); mientras que Caballero-Zamora et al. (2015) reportan que no fue posible 
estimar esta correlación en presencia de SMB, debido a la pérdida de estructura de información derivada de la alta mortalidad en la población. Por otro lado, no hay información sobre cómo se modifican estas ra para peso y supervivencia a través de diferentes ambientes en la producción de camarón. Por lo anterior, es importante estimar estos parámetros genéticos ( $h^{2}$ y $\mathrm{r}_{\mathrm{G}}$ ) en presencia o ausencia del SMB para el diseño óptimo de los PMG.

Por lo tanto, el objetivo de este estudio fue estimar los efectos de IGA para PC y SC en dos ambientes comerciales (presencia o ausencia de brote natural del SMB), en dos líneas genéticas de camarón blanco del Pacífico (Penaeus vannamei), una seleccionada para crecimiento y otra con antecedentes de resistencia al SMB.

\section{MATERIAL Y MÉTODOS}

Los datos fueron obtenidos de una compañía de producción de larvas de camarón, localizada en el noroeste de México. Los registros incluyeron camarones del ciclo de producción 2016. Los camarones fueron criados bajo condiciones comerciales en tres estanques: dos estanques estuvieron ubicados en dos granjas establecidas en el estado de Sonora (Kino y Marea Alta); los cuales presentaron un brote natural de SMB (SMBpresencia), donde el diagnóstico se realizó a partir de la sintomatología y cambios macroscópicos típicos del SMB (Stentiford y Lightner, 2011), durante el ciclo de producción y confirmado mediante análisis de PCR. El tercer estanque se localizó en la comunidad de Los Pozos, Sinaloa (Pozos), donde se realizaron procedimientos estrictos de bioseguridad y no se detectaron síntomas de SMB, ni hubo diagnóstico positivo por PCR (SMB-ausencia).

Se utilizó una línea seleccionada desde 1998 para crecimiento y SC (CRE), y otra línea con antecedentes de resistencia a SMB (RES). Las familias consideradas de cada línea en este estudio tuvieron un máximo de $25 \%$ de genes de la otra línea (Gallaga-Maldonado et al., 2020), y se analizaron de manera independiente. Para la línea CRE se analizaron 7,679 registros procedentes de apareamientos entre 49 padres y 69 madres (familias). Para la línea RES se usaron 9,519 registros con la progenie de 63 padres y 91 madres (familias). La razón de hembras por macho fue de 1.4 en ambas líneas. La información del pedigrí incluyó animales nacidos desde 2002 para CRE y desde 2014 para RES.

\section{Origen y desarrollo de las líneas genéticas}

Las líneas CRE y RES fueron formadas en 1998 y 2014 respectivamente. La línea CRE fue producida usando camarones provenientes de México, Venezuela, Colombia, Estados Unidos, y Ecuador. La línea RES está compuesta por camarones con antecedentes de resistencia a SMB procedentes de Ecuador, Panamá y Estados Unidos y desde 2014. Una descripción más específica de la creación de las líneas genéticas puede encontrarse en Gallaga-Maldonado et al. (2020) y Campos-Montes et al. (2020). 


\section{Manejo de familias}

Las familias fueron producidas mediante inseminación artificial, utilizando una relación de un macho por cada dos hembras para la formación de familias de medios hermanos. Las hembras inseminadas desovaron en tanques individuales para el conteo de nauplios por familia (hermanos completos); siendo descartados aquellos desoves con menos de 25,000 nauplios para la siguiente etapa. La alimentación en las fases larvarias se basó en micro-pellets comerciales con $40 \%$ a $50 \%$ de proteína y $8 \%$ a $10 \%$ de grasa, microalgas Chaetoceros, Spirulina spp, y Artemia spp., y la constitución de la dieta se adecuó a cada estadio. Las familias de hermanos completos se mantuvieron en el mismo estanque hasta que fueron marcadas alrededor de los 60 días de edad (pesando entre 2 y 3 gramos), mediante elastómeros de colores (Northwest Marine Technology ${ }^{\mathrm{TM}}$ ), en el último segmento abdominal del camarón y cuya combinación de colores funcionó como identificación familiar.

\section{Manejo de los estanques de crecimiento}

Diez días posteriores al marcaje, se sembraron 36 camarones en promedio por familia en cada estanque. Los Estaques de Sonora (SMB-presencia) eran de arena de 0.20 ha, con una columna de agua de $1.4 \mathrm{~m}$, a una temperatura promedio de $32^{\circ} \mathrm{C}$ y una salinidad promedio de $33 \mathrm{gL}^{-1}$. La tasa de intercambio diario de agua varió de $5 \%$ a $20 \%$. El alimento ofrecido contó con un porcentaje de proteína entre el 34 y $40 \%$ a razón de 3\% de la biomasa total en el estanque. La densidad de siembra en ambos estanques fue de 16 organismos $/ \mathrm{m}^{2}$. En Sinaloa (SMB-ausencia) se usó un estanque de concreto de $4 \mathrm{x}$ $16 \mathrm{~m}$, con una columna de agua de $2 \mathrm{~m}$ y una densidad de siembra de $70 \mathrm{~m}^{2}$. La temperatura del agua se mantuvo a $30^{\circ} \mathrm{C}$ con una salinidad de $35 \mathrm{gL}-1$, aireación constante y una tasa de intercambio de agua diario de $4 \%$ a $5 \%$. La cantidad de alimentación diaria ofrecida (35\% - 40\% de proteína); se calculó como el $6 \%$ de su biomasa.

\section{Recolección de datos para peso corporal a los 130 días de edad y supervivencia de 70 a 130 días}

Posterior a 70 de la siembra se recuperó la totalidad de los organismos de los estanques, y de cada uno se identificó la familia de origen, el sexo y peso corporal. Se eliminó la información de individuos con deformidades, sin identificación confiable o sexo indefinido. Para la estimación de la SC se consideró a los animales recuperados al final del periodo como vivos (1) y los animales no recuperados, considerando la diferencia entre los organismos vivos de cada familia y los sembrados, como muertos (0). 


\section{Análisis de la información}

Para comparar el comportamiento productivo entre ambas líneas en ambos escenarios (SMB-presencia y SMB-ausencia), se consideró el siguiente modelo lineal:

$$
y_{i j k}=\mu+L_{i}+S_{j}+L_{i j}+e_{i j k}
$$

Donde, $\boldsymbol{y}_{\mathrm{ijk}}$ es el vector de observaciones de PC o SC, $\boldsymbol{\mu}$ es la media de la población para la variable de interés (SC o PC), $L_{i}$ es el efecto de la i-esima línea (CRE, RES), $\mathbf{S}_{j}$ es el efecto del j-esimo ambiente (SMB-presencia, SMB-ausencia), $\mathbf{L S}_{\mathrm{ij}}$ es el efecto de la interacción entre la línea y estado de salud del estanque, y $e_{i j k} \sim N\left(0, \sigma^{2}\right)$. El sexo y el estanque también se incluyeron en PC. Para determinar diferencias entre combinaciones de línea y ambiente se usó una prueba de Tukey $(\alpha=0.05)$.

Los parámetros genéticos para PC y SC fueron estimados para cada línea, usando un modelo animal y máxima verosimilitud restringida, con el software ASReml. Considerando los criterios de aproximación de una distribución binomial a una distribución normal (Schader y Schmid, 1989; Emura y Yu-Ting, 2018) se asumió normalidad en el análisis de SC; el modelo usado fue:

$$
y=X \beta+Z u+W f+\varepsilon
$$

Donde, $\boldsymbol{y}$ es el vector de observaciones de (PC o SC) de ambos ambiente, $\boldsymbol{\beta}$ es el vector de efectos fijos de cada característica, $\mathbf{u}$ es el vector de efectos genéticos aditivos aleatorios del animal, $\mathbf{u} \sim \mathrm{MVN}(0, \mathbf{G})$, donde $\mathbf{G}=\mathbf{V} \otimes \mathbf{A}$, donde $\mathbf{V}$ es una matriz simétrica que contiene las (co) varianzas entre los efectos de los animales de las misma familias para las características en los dos ambientes, y A es una matriz de relaciones aditivas; $\otimes$ es el producto Kronecker, residuales ambientales, $f$ es el vector desconocido del efecto común de familia para todas las características, $\mathbf{f} \sim \mathrm{MVN}(0, \mathbf{F})$; donde, $\mathbf{F}=\mathbf{C} \otimes \mathbf{I}$, donde, C es una matriz de co(varianzas) de los efectos de ambiente común de familia, sólo para PC, e I es una matriz de identidad de orden apropiado, y $\varepsilon$ es el vector de efectos aleatorios, $\boldsymbol{\varepsilon} \sim \operatorname{MVN}(0, \mathbf{R})$, donde, $\mathbf{R}=\mathbf{E} \otimes \mathbf{I}$, donde, $\mathbf{E}$ es la matriz de co(varianzas)de los efectos residuales que contiene las covarianzas entre las dos características, e I es una matriz de identidad de orden apropiado, con $\boldsymbol{\sigma}^{2} \mathrm{e}$ como la varianza residual.

Por último, $\mathbf{X}, \mathbf{Z}$, y $\mathbf{W}$ son matrices de incidencia conocidas que relacionan las observaciones con los efectos fijos (que variaron dependiendo de la característica analizada), los efectos genéticos del animal y los efectos de ambiente común de familia, respectivamente. Las correlaciones genéticas entre ambas características en la combinación línea-estanque se estimaron con ASReml, usando modelos bivariados y con el mismo modelo, pero considerando el vector $\boldsymbol{y}$ de información de PC y SC. No se usaron restricciones en la estructura de covarianzas y los efectos de ambiente común de familia, fueron considerados independientes. 
En la estimación de los parámetros genéticos para el PC, los efectos fijos incluidos en el modelo, fueron: sexo, edad a la cosecha lineal y cuadrática, adicionalmente en el caso de SMB-presencia; se incluyó el efecto de estanque (Kino y Marea alta). En cuanto a la SC para los estanques afectados, el único efecto fijo considerado fue el de estanque en SMB-presencia; mientras que en el ambiente SMB-ausencia no se consideró ningún efecto fijo.

La varianza fenotípica para cada característica, se estimó como la suma de los componentes de varianza de los efectos aleatorios (genético del animal y común de familia). La $\mathrm{h}^{2}$ se estimó como la proporción de la varianza fenotípica que se debe a la varianza genética aditiva, y la ra se estimó como la covarianza dividida en el producto de las correspondientes desviaciones estándar. La significancia estadística de los parámetros estimados se basó en los intervalos de confianza (95\%), construidos con sus errores estándar, asumiendo normalidad. La existencia de IGA se determinó cuando rG entre ambientes fue menor que 0.80 (Sae-Lim et al., 2016).

Finalmente, para analizar si el comportamiento de las características entre líneas genéticas fue similar, se realizó una comparación de las ra estimadas en cada línea (Nguyen et al., 2016), dicha comparación se realizó por medio de una transformación Z de Fisher (Rosenthal et al., 1992) implementada en el paquete "Cocor" en R (Diedenhofen y Musch, 2014), una prueba de significancia para la diferencia entre 2 correlaciones, basada en grupos dependientes con 1 variable en común. Para una mejor comprensión, se presenta un esquema de las correlaciones genéticas estimadas por línea en la figura 1.

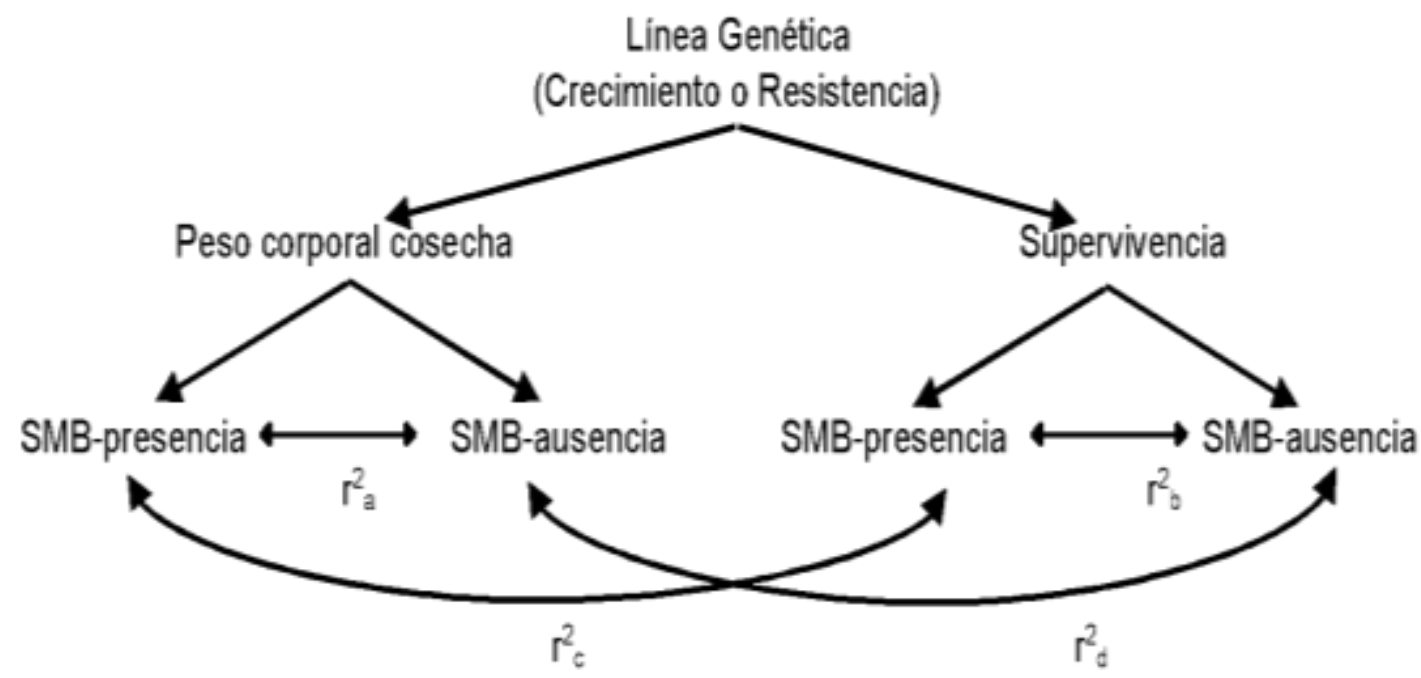

Figura 1. Esquema de las correlaciones genéticas estimadas en el estudio por línea genética $r^{2}$ a y $r^{2} b=$ Correlación genética entre ambientes (SMB-presencia y SMB-ausencia) dentro de cada característica. $r^{2} c=$ Correlación genética entre los ambientes SMB-presencia de las dos características. $r^{2} d$ = Correlación genética entre los ambientes SMB-ausencia de las dos características 


\section{RESULTADOS Y DISCUSIÓN}

\section{Comparación del comportamiento productivo entre líneas}

Las estadísticas descriptivas para PC y SC en cada línea genética (CRE y RES), dentro de ambiente (SMB-presencia, SMB-ausencia) se muestran en el cuadro 1. Al mismo tiempo, las figuras 2 y 3 muestran las LSM de ambas líneas (CRE y RES), a través de los ambientes para PC y SC. Estos resultados muestran diferencias en SC, donde la línea CRE tiene una baja SC en SMB-presencia; mientras que los camarones de la línea RES poseen una menor SC en SMB-presencia. Adicionalmente, existe interacción línea por ambiente $(\mathrm{P}<0.0001)$ en las dos características; dichas interacciones de línea por ambiente resaltan la importancia de considerar la probabilidad de ocurrencia de la enfermedad SMB, cuando se elige la línea en el programa de mejoramiento genético (Sae-Lim et al., 2016).

Cuadro 1. Número de individuos (n) y medias mínimo cuadráticas para peso corporal y tasa de supervivencia a la cosecha en la línea de crecimiento y en la línea de Resistencia, en presencia y ausencia del Síndrome de Mancha Blanca

\begin{tabular}{|c|c|c|c|c|c|}
\hline \multirow[b]{2}{*}{ Ambiente } & \multirow[b]{2}{*}{ Line } & \multicolumn{2}{|c|}{ Peso corporal (g) } & \multicolumn{2}{|c|}{ Tasa de Supervivencia } \\
\hline & & $\mathbf{n}$ & $\begin{array}{l}\text { LSM } \pm \\
\text { ee }\end{array}$ & $\mathbf{n}$ & LSM \pm ee \\
\hline \multirow{2}{*}{ SMB-presencia } & RES & 2,524 & $12.80 \pm 0.07^{a}$ & 6,414 & $0.45 \pm 0.01^{a}$ \\
\hline & GRO & 294 & $8.75 \pm 0.06^{b}$ & 5,494 & $0.06 \pm 0.01^{b}$ \\
\hline \multirow{2}{*}{ SMB-ausencia } & RES & 2,838 & $11.91 \pm 0.17^{c}$ & 3,105 & $0.82 \pm 0.01^{c}$ \\
\hline & GRO & 1,926 & $13.75 \pm 0.05^{d}$ & 2,185 & $0.88 \pm 0.01^{d}$ \\
\hline
\end{tabular}

LSM: Medias mínimo cuadráticas, ee: Error estándar.

*Los diferentes literales dentro de las columnas indican diferencias estadísticamente significativas TUKEY $(\alpha=0.05)$.

\section{Heredabilidades para peso corporal a la cosecha}

Las heredabilidades para PC en ambas líneas se muestran en el cuadro 2. Los efectos de ambiente común de familia fueron 0.05 en ambas líneas. La inclusión de estos efectos en todos los modelos redujo la estimación de la varianza aditiva, acorde a lo presentado por otros autores (Campos-Montes et al., 2013; Montaldo et al., 2013). Las heredabilidades en SMB-ausencia fueron consistentes con los presentados por autores como Tan et al. (2017), Trang et al. (2019) y Ren et al. (2020), a pesar de que éste último no consideró efectos de ambiente común de familia en su modelo; sin embargo, estos estimados de heredabilidad fueron mayores que los reportados por otros autores ( $\mathrm{Li}$ et al., 2015; Zhang et al., 2017; Yuan et al., 2018). Por otro lado, las heredabilidades para SMB-presencia son similares a los estimados por Caballero-Zamora et al. (2015), quienes también usaron datos provenientes de un brote natural de SMB. 


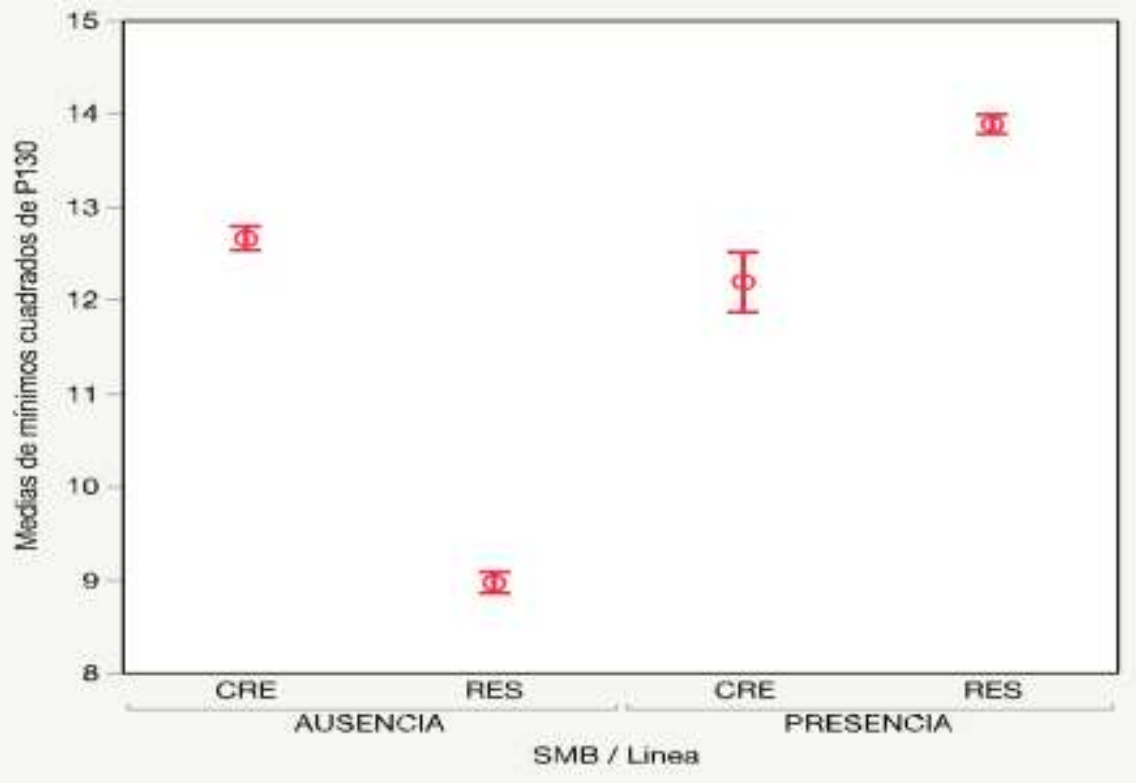

Figura 2. Medias mínimo cuadráticas para peso corporal a la cosecha en las líneas de crecimiento y resistencia a SMB, en presencia y ausencia de SMB en $P$. vannamei

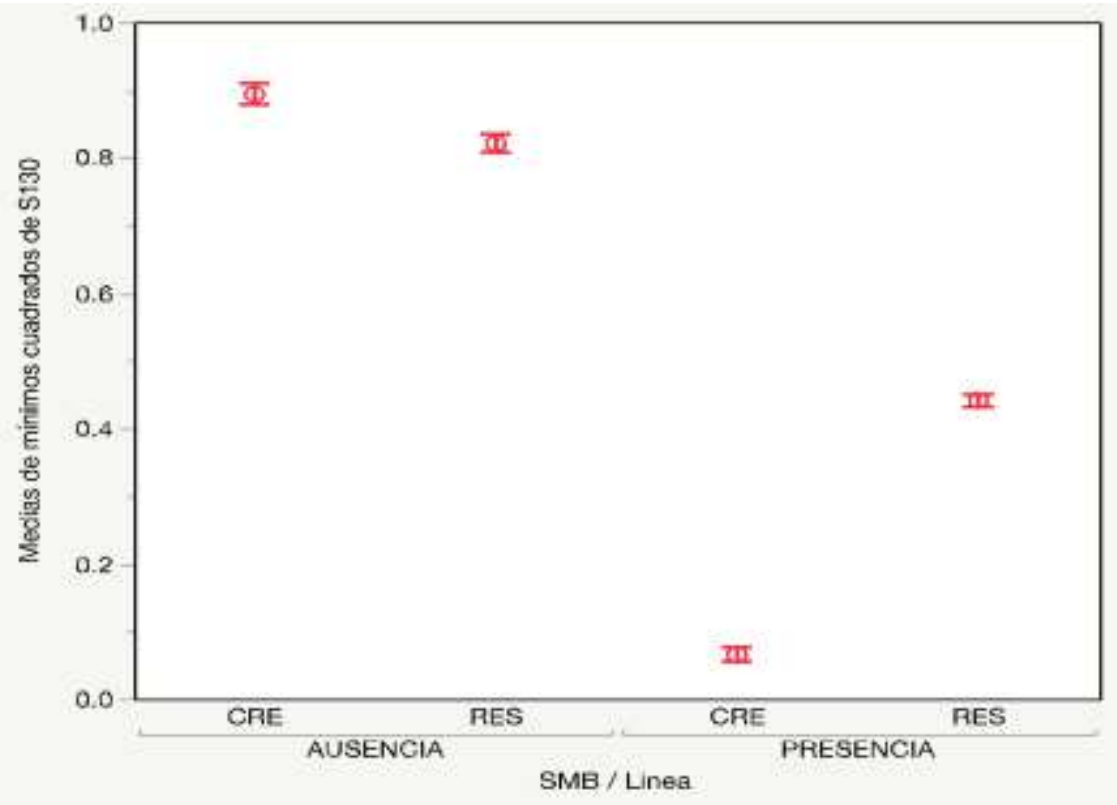

Figura 3. Medias mínimo cuadráticas para supervivencia a la cosecha en las líneas de crecimiento y resistencia a SMB, en presencia y ausencia de SMB en $P$. vannamei

En la línea CRE, la diferencia entre las heredabilidades para SMB-presencia $(0.05 \pm 0.16)$ y SMB-ausencia $(0.35 \pm 0.15)$, puede ser un indicador de heterogeneidad de varianzas (Sae-Lim et al., 2016); pero en este caso, además de cambios en la varianza genética aditiva, la fuente de esta heterocedasticidad puede estar contenida en cambios en la varianza ambiental por la sensibilidad micro ambiental de los individuos. En el caso de 
SMB-presencia, es importante mencionar que la poca precisión en el estimado de $\mathrm{h}^{2}$ puede deberse a la estructura de datos resultante de la alta mortalidad. En la línea RES, los estimados de heredabilidad para PC fueron mayores que para CRE; además de tener mejores precisiones. Por otro lado, el estimado de $h^{2}$ en SMB-presencia $(0.26 \pm 0.07)$ es menor que en SMB-ausencia $(0.49 \pm 0.08)$, al igual que en la línea CRE, y los estimadores de esta línea fueron consistentes con otros autores (Luan et al., 2015; Sui et al., 2016; Zhang et al., 2017 y Yuan et al., 2018).

Cuadro 2. Heredabilidades y correlaciones genéticas aditivas para peso corporal en las líneas de crecimiento y resistencia por ambiente*

\begin{tabular}{llcr}
\hline & Ambiente & SMB-presencia & SMB-ausencia \\
\hline \multirow{2}{*}{ Línea de Crecimiento } & SMB-presencia & $0.05 \pm 0.16$ & $\mathbf{- 0 . 1 7 \pm \mathbf { 0 . 6 0 }}$ \\
& SMB-ausencia & & $0.35 \pm 0.15$ \\
& SMB-presencia & $0.26 \pm 0.07$ & $\mathbf{0 . 8 9} \pm \mathbf{0 . 0 9}$ \\
Línea de Resistencia & SMB-ausencia & & $0.49 \pm 0.08$ \\
\hline $\begin{array}{l}\text { *Estimados mediante análisis bivariados. } \\
\text { en negrilla }\end{array}$ & Las correlaciones genéticas se muestran
\end{tabular}

Las variaciones en los estimadores de heredabilidad para PC pueden ser resultado de la sensibilidad micro ambiental de los individuos (Sae-Lim et al., 2016). Dado lo anterior, es importante tener en cuenta que estos cambios en la heredabilidad pueden alterar la precisión de la predicción de la respuesta a la selección.

\section{Correlaciones genéticas para PC}

La rG estimada para los PC entre ambientes en la línea CRE fue negativa y no significativa $(P>0.05)(-0.17 \pm 0.60)$; aunque en las estimaciones con modelos preliminares (datos no presentados) fue consistentemente negativa. El valor poco preciso de la estimación puede ser consecuencia de los bajos valores de la varianza genética aditiva de PC en SMS-presencia, lo cual complica la evaluación de efectos de IGA en esta línea; mientras que en la línea RES, esta correlación no fue diferente de $1(P>0.05)$ (cuadro 2); señalando que los efectos genéticos aditivos para PC son muy similares en los dos ambientes (SaeLim et al., 2016). En otras palabras, no existe efecto de IGA en RES para PC. Considerando que ambas líneas estuvieron en las mismas condiciones ambientales de manejo y expuestas al mismo patógeno (SMB), es posible considerar que las diferencias en los estimadores de ambas líneas son resultado de la baja tasa de SC de la línea CRE. En varios estudios no se detectó IGA al considerar condiciones ambientales, como la densidad de siembra (Campos-Montes et al., 2009; Tan et al., 2017) o localidad de cultivo (Sui et al., 2016). Otros trabajos reportan posibles efectos de IGA para PC, considerando los valores puntuales de los estimadores de Rg; sin embargo, los errores estándar de 
éstas, no permiten definirlas como significativamente diferentes de uno o cero (CaballeroZamora et al., 2015; Li et al., 2015; Nguyen et al., 2020).

\section{Heredabilidades para supervivencia a la cosecha en ambas líneas genéticas}

Las heredabilidades y las correlaciones genéticas aditivas para SC en las líneas genéticas se muestran en el cuadro 3. En cuanto a los efectos comunes de familia, estos fueron considerados independientes entre ambientes para cada característica, y variaron entre 0.02 y 0.04. Las heredabilidades estimadas para SC en SMB-presencia fueron 0.01 y 0.11 para las CRE y RES respectivamente; los anteriores son similares a las reportadas por otros autores en presencia de SBM (Gitterle et al., 2005a; Caballero-Zamora et al., 2015); sin embargo, fueron menores que los estimados por Li et al. (2015) y Trang et al. (2019); ambos en desafíos controlados a SMB. Los resultados del modelo de SC fueron consistentes con estimaciones, usando modelos univariados, considerando una distribución binomial (resultados no mostrados).

Cuadro 3. Heredabilidades y correlaciones genéticas aditivas para supervivencia en las líneas de crecimiento y resistencia por ambiente*

\begin{tabular}{llrr}
\hline & Ambiente & SMB-presencia & SMB-ausencia \\
\hline \multirow{2}{*}{ Línea de Crecimiento } & SMB-presencia & $0.01 \pm 0.02$ & $\mathbf{0 . 0 0}$ \\
& SMB-ausencia & & $0.01 \pm 0.03$ \\
& SMB-presencia & $0.11 \pm 0.06$ & $\mathbf{0 . 1 0 \pm 0 . 4 0}$ \\
Línea de Resistencia & SMB-ausencia & $0.06 \pm 0.04$ \\
\hline $\begin{array}{l}\text { * Estimados usando modelos bivariados } \\
\text { Las correlaciones genéticas se muestran en negrilla }\end{array}$
\end{tabular}

Las heredabilidades para SC en SMB-ausencia fueron $0.01 \pm 0.03$ en CRE y $0.06 \pm 0.04$ en RES concordantes con otros estimadores para supervivencia general (CamposMontes et al., 2013; Zhang et al., 2017; Ren et al., 2020), pero menores a los presentados por Lu et al. (2017), Luan et al. (2020), y Tan et al. (2017), donde no se incluyó el efecto de ambiente común de familia, lo cual podría haber generado una sobrestimación de los parámetros genéticos.

Las heredabilidades en CRE fueron esencialmente cero en ambos ambientes, $0.01 \pm 0.02$ en SMB-presencia y $0.02 \pm 0.03$ en SMB-ausencia, lo cual, representa mínimas posibilidades de avance genético por selección para esta característica en ambos escenarios, lo cual es consistente con otros autores (Gitterle et al., 2005a; CamposMontes et al., 2013; Li et al., 2015; Lu et al., 2017). El avance mínimo por selección podría estar relacionado con la dificultad en la estimación a causa de la tasa de mortalidad por parte de los modelos estadísticos (Vehviläinen et al., 2008), una proporción genética muy 
baja en la expresión de la supervivencia, o posiblemente, el daño en la estructura de relaciones genéticas de familia cuando SMB estuvo presente.

Las heredabilidades estimadas para SC en RES fueron $0.11 \pm 0.06$ y $0.06 \pm 0.04$, para SMB-presencia y SMB-ausencia, respectivamente (cuadro 3); las cuales no fueron diferentes estadísticamente a las estimadas para la línea CRE. La $\mathrm{h}^{2}$ para SC en SMBpresencia fue superior la estimada por Caballero-Zamora et al. (2015) 0.06 y a la reportada por Gitterle et al. (2005b), que calculó valores entre 0.03 a 0.07 . La h ${ }^{2}$ para SMB-ausencia fue concordante con otros estimadores para supervivencia general en la misma especie (Campos-Montes et al., 2013; Zhang et al., 2017; Ren et al., 2020).

Las heredabilidades de SC para ambos ambientes fueron consistentes en ambas líneas (cuadro 3), sugiriendo que no existe una compresión de la varianza aditiva, en las líneas, asociada al ambiente (Sae-Lim et al., 2016). Las correlaciones genéticas entre SC en SMB-presencia y SMB-ausencia no fueron diferentes de cero $(P>0.05)$, coincidiendo con lo reportado por Vehviläinen et al. (2010) en otra especie acuática, lo cual puede proponer que las supervivencias en ambos ambientes son características independientes, como lo sugiere Thoa et al. (2015).

\section{Correlaciones genéticas entre peso corporal y supervivencia a la cosecha}

Las correlaciones genéticas por ambiente entre PC y SC se muestran en el cuadro 4. Para la estimación de estas correlaciones el efecto común de familia se consideró independiente entre ambientes.

Cuadro 4. Correlaciones genéticas dentro de líneas (Crecimiento y Resistencia) entre peso corporal a la cosecha (PC) y supervivencia a la cosecha para ambientes en presencia o ausencia de SMB

\begin{tabular}{lrr}
\hline & SMB-presencia & SMB-ausencia \\
\hline Línea de crecimiento & $n e^{*}$ & $-0.09 \pm 0.92$ \\
Línea de resistencia & $0.04 \pm 0.16$ & $0.57 \pm 0.20$ \\
\hline${ }^{*}$ No estimable & &
\end{tabular}

La correlación genética entre las dos características no pudo ser estimada en SMBpresencia en la línea CRE, posiblemente por la afectación en la estructura de la información asociada con la alta mortalidad presentada en esa línea; en tanto que, para la línea RES, no fue diferente de cero $(0.04 \pm 0.16)$, concordando con los resultados de Caballero-Zamora et al. (2015). En el caso de SMB-ausencia para la línea CRE, la ra no fue diferente de cero $(-0.09 \pm 0.92)$, a diferencia de la estimada en la línea RES que fue de $0.57 \pm 0.20$, la cual fue consistente con la estimada por Campos-Montes et al. (2013), en estanques comerciales, en ausencia de SMB $(0.56 \pm 0.10)$. La rg estimada en la línea RES fue mayor que la reportada por Yuan et al. (2018), Gitterle et al. (2005a), y Zhang et al. (2017). Las diferencias entre la correlación genética en RES pueden ser indicador de cambios en los componentes de varianza posiblemente asociados a IGA, a su vez 
relacionada con las covarianzas correspondientes, lo que tendría implicaciones en la respuesta a la selección correlacionada (Sae-Lim et al., 2016).

Algunos autores resaltan la importancia de la creación de líneas genéticas en acuicultura (Nguyen et al., 2016), los resultados de este estudio sugieren que los índices de selección para PC deben tener en cuenta la línea genética usada en el programa de mejoramiento genético. Por otro lado, la estimación de los parámetros genéticos relacionada a PC debe considerar la presencia de enfermedades endémicas, como es el caso de SMB en el cultivo de camarón y visualizar la SC en presencia y ausencia de SMB como características independientes, en ambas líneas genéticas.

Además de los cambios en las heredabilidades y correlaciones genéticas en ambas líneas, la productividad en éstas fue diferente en los ambientes estudiados, lo cual, podría interpretarse como un indicador de plasticidad fenotípica, que puede ser común en organismos marinos como lo sugiere Munday, (2013), y puede entenderse como la expresión de diferentes fenotipos en individuos con el mismo genotipo, pero bajo diferentes condiciones ambientales (Munasinghe y Seneviratha, 2015). En consecuencia, dicho efecto de plasticidad fenotípica debería ser considerado en los programas de mejoramiento genético de camarón, procurando que los ambientes de desempeño a analizar sean lo más parecidos a las condiciones de producción (Nguyen et al., 2016).

\section{CONCLUSIONES}

Los resultados del modelo lineal sugieren diferencias entre las líneas, tanto para peso corporal como para supervivencia a través de los ambientes; sin embargo, las estimaciones de las correlaciones genéticas no permiten considerar efectos de IGA dentro de línea en ambas características, lo que indicaría que son independientes. Además, las correlaciones genéticas entre las características de la línea de resistencia proponen tratarlas como variables independientes, cuando SMB está presente en el ambiente.

\section{LITERATURA CITADA}

CABALLERO-ZAMORA A, Montaldo HH, Campos-Montes GR, Cienfuegos-Rivas EG, Martínez-Ortega A, Castillo-Juárez H. 2015. Genetic parameters for body weight and survival in the Pacific White Shrimp Penaeus (Litopenaeus) vannamei affected by a White Spot Syndrome Virus (WSSV) natural outbreak. Aquaculture. 447:102-107. ISSN: 00448486. https://doi.org/10.1016/j.aquaculture.2014.08.028

CAMPOS-MONTES GR, Montaldo HH, Martínez-Ortega A, Castillo-Juárez H. 2009. Genotype by environment interaction effects for body weight at 130 days of age in the Pacific white shrimp [Penaeus (Litopenaeus) vannamei]. Veterinaria México. 40(3):255267. ISSN: 2448-6760.

https://www.medigraphic.com/cgi-

bin/new/resumenl.cgi?IDARTICULO=22647 
CAMPOS-MONTES GR, Montaldo HH, Martínez-Ortega A, Jiménez AM, Castillo-Juárez H. 2013. Genetic parameters for growth and survival traits in Pacific white shrimp Penaeus (Litopenaeus) vannamei from a nucleus population undergoing a two-stage selection program. Aquaculture International. 21:299-310. ISSN: 1573-143X. https://doi.org/10.1007/s10499-012-9553-1

CAMPOS-MONTES GR, Caballero-Zamora A, Montaldo HH, Montoya-Rodríguez L, Rodríguez-Sala BGG, Rodríguez SAS, Martínez-Ortega A, Quintana-Casares JC, Castillo-Juárez H. 2020. Genetic (co) variation in resistance of Pacific white shrimp Litopenaeus vannamei to acute hepatopancreatic necrosis disease (AHPND) and white spot syndrome virus (WSSV) in challenge tests. Aquaculture. 520:734994. ISSN: 00448486. https://doi.org/10.1016/j.aquaculture.2020.734994

DIEDENHOFEN B, MUSCH J. 2015. cocor: A comprehensive solution for the statistical comparison of correlations. PloS one. 10(4):e0121945. ISSN: 1932-6203. https://doi.org/10.1371/journal.pone.0121945

EMURA T, Liao YT. 2018. Critical review and comparison of continuity correction methods: The normal approximation to the binomial distribution. Communications in Statistics-Simulation and Computation. 47(8):2266-2285. ISSN: 0361-0918. https://doi.org/10.1080/03610918.2017.1341527

GALLAGA-MALDONADO EP, Montaldo HH, Castillo-Juárez H, Campos-Montes GR, Martínez-Ortega A, Quintana-Casares JC, Montoya-Rodríguez L, Betancourt-Lozano M, Lozano-Olvera R, Vázquez-Peláez C. 2020. Crossbreeding effects for White Spot Disease resistance in challenge tests and field pond performance in Pacific white shrimp Litopenaeus vannamei involving susceptible and resistance lines. Aquaculture. 516:734527. ISSN: 0044-8486. https://doi.org/10.1016/j. aquaculture.2019.734527

GITTERLE T, Rye M, Saltec R, Cock J, Johansen H, Lozano C, Suárez J, Gjerde B. 2005a. Genetic (co)variation in harvest body weight and survival in (Penaeus (Litopenaeus) vannamel) under standard commercial conditions. Aquaculture. 243:8392. ISSN: 0044-8486. https://doi.org/10.1016/j. aquaculture.2004.10.015

GITTERLE T, Saltec R, Gjerde B, Cock J, Johansen H, Salazar M, Lozano C, Rye M. 2005b. Genetic (co)variation in resistance to White Spot Syndrome Virus (WSSV) and harvest weight in Penaeus (Litopenaeus) vannamei. Aquaculture. 246:139-149. ISSN: 0044-8486. https://doi.org/10.1016/j.aquaculture.2005.02.011

GITTERLE T, Odegard J, Gjerde B, Rye M, Salte R. 2006a. Genetic parameters and accuracy of selection for resistance to white spot syndrome virus (WSSV) in Penaeus (Litopenaeus) vannamei using different statistical models. Aquaculture. 251:210-218. ISSN: 0044-8486. https://doi.org/10.1016/j.aquaculture.2005.06.008 
GITTERLE T, Gjerde B, Cock J, Salazar M, Rye M, Vidal O, Lozano C, Erazo C, Salte R. 2006b. Optimization of experimental infection protocols for the estimation of genetic parameters of resistance to White Spot Syndrome Virus (WSSV) in Penaeus (Litopenaeus) vannamei. Aquaculture. 261:501-509. ISSN: 0044-8486. https://doi.org/10.1016/j.aquaculture.2006.07.017

HERNÁNDEZ-LLAMAS A, Cabanillas-Ramos J, Magallon-Barajas FJ. 2016. Estimating impact of white spot disease on economic risk in semi-intensive shrimp farms in Mexico: the case of the State of Sinaloa. Reviews in Aquaculture. 8(2):111-120. ISSN: 1753-5123. https://doi.org/10.1111/raq.12084

HUANG Y, Yin Z, Weng S, He J, Li S. 2012. Selective breeding and preliminary comercial performance of Penaeus vannamei for resistance to resistance to White spot síndrome virus (WSSV). Aquaculture. 364-365:111-117. ISSN: 0044-8486. https://doi.org/10.1016/j.aquaculture.2012.08.002

IBARRA AM, Famula TR. 2008. Genotype by environment interaction for adult body weights of shrimp Penaeus vannamei when grown at low and high densitie. Genetics Selection Evolution. 40(5):541. ISSN: 0999-193X. https://doi.org/10.1186/1297-9686-405-541

KLINGER D, Naylor R. 2012. Searching for solutions in aquaculture: charting a sustainable course. Annual Review of Environment and Resources. 37:247-276. ISSN: 1543-5938. https://doi.org/10.1146/annurev-environ-021111-161531

LI W, Luan S, Luo K, Sui J, Xu X, Tan J, Kong J. 2015. Genetic parameters and genotype by environment interaction for cold tolerance, body weight, and survival of the Pacific white shrimp Penaeus vannamei at different temperatures. Aquaculture. 441:8-15. ISSN: 00448486. https://doi.org/10.1016/j.aquaculture.2015.02.013

LU X, Luan S, Cao B, Sui J, Dai P, Meng X, Luo K, Kong J. 2017. Heterosis and heritability estimates for the survival of the Pacific white shrimp (Litopenaeus vannamei) under the commercial scale ponds. Acta Oceanologica Sinica. 36(2):62-68. ISSN: 0253-505X. https://doi.org/10.1007/s13131-016-0942-6

LUAN S, Luo K, Chai Z, Cao B, Meng X, Lu X, Liu N, Xu S, Kong J. 2015. An analysis of indirect genetic effects on adult body weight of the Pacific white shrimp Litopenaeus vannamei at low rearing density. Genetics Selection Evolution. 47:95. ISSN: 0999-193X. https://doi.org/10.1186/s12711-015-0164-y

LUAN S, Qiang G, Cao B, Luo K, Meng X, Chen B, Kong J. 2020. Feed competition reduces heritable variation for body weight in Litopenaeus vannamei. Genetics Selection Evolution. 52:45. ISSN: 0999-193X. https://doi.org/10.1186/s12711-020-00565-3 
MONTALDO HH, Castillo-Juárez H, Campos-Montes G, Pérez-Enciso M. 2013. Effect of the data family structure, tank replication and the statistical model, on the estimation of genetic parameters for body weight at 28 days of age in the Pacific white shrimp (Penaeus (Litopenaeus) vannamei Boone, 1931). Aquaculture Research. 44(11):1715-1723. ISSN: 1355-557X. https://doi.org/10.1111/j.1365-2109.2012.03176.x

MUNASINGHE DHN, Senevirathna JDM. 2015. Phenotypic plasticity and genetic variation of two wild populations of green tiger shrimp (Penaeus semisulcatus - De Haan, 1844). International Journal of Marine Science. 5:1-8. ISSN: 1927-6648. http://www.aquapublisher.com/index.php/ijms/article/view/1561

MUNDAY PL, Warner RR, Monro K, Pandolfi JM, Marshall DJ. 2013. Predicting evolutionary responses to climate change in the sea. Ecology letters. 16(12):1488-1500. ISSN: 1461-023X. https://doi.org/10.1111/ele.12185

NGUYEN NH. 2016. Genetic improvement for important farmed aquaculture species with a reference to carp, tilapia and prawns in Asia: achievements, lessons and challenges. Fish and Fisheries. 17(2):483-506. ISSN: 1467-2979. https://doi.org/10.1111/faf.12122

NGUYEN NH, Ninh NH, Hung NH. 2020. Evaluation of two genetic lines of Pacific White leg shrimp Liptopenaeus vannamei selected in tank and pond environments. Aquaculture. 516:734522. ISSN: 0044-8486. https://doi.org/10.1016/j.aquaculture.2019.734522

ØDEGÅRD J, Baranski M, Gjerde B, Gjedrem T. 2011. Methodology for genetic evaluation of disease resistance in aquaculture species: challenges and future prospects. Aquaculture research. 42:103-114. ISSN: 1355-557X. https://doi.org/10.1111/j.1365-2109.2010.02669.x

REN S, Prentis P, Mather PB, Li Y, Tang B, Hurwood DA. 2020. Genetic parameters for growth and survival traits in a base population of Pacific white shrimp (Litopenaeus vannamel) developed from domesticated strains in China. Aquaculture. 523:735-148. ISSN: 0044-8486. https://doi.org/10.1016/j.aquaculture.2020.735148

ROSENTHAL R, Rubin DB, Meng XL. 1992. Comparing correlated correlation coefficients. Psychological bulletin. 111(1):172-175. ISSN: 0033-2909. APA Psycnet http://nrs.harvard.edu/urn-3:HUL.InstRepos:11718225

SAE-LIM P, Gjerde B, Nielsen HM, Mulder H, Kause A. 2016. A review of genotype byenvironment interaction and micro-environmental sensitivity in aquaculture species. Reviews in Aquaculture. 8(4):369-393. ISSN: 1753-5123. https://doi.org/10.1111/raq.12098

SCHADER M, Schmid F. 1989. Two rules of thumb for the approximation of the binomial distribution by the normal distribution. The American Statistician. 43(1):23-24. ISSN: 0003-1305. https://doi.org/10.1080/00031305.1989.10475601 
STENTIFORD GD, Lightner DV. (2011). Cases of white spot disease (WSD) in European shrimp farms. Aquaculture. 319(1-2):302-306. ISSN: 0044-8486. https://doi.org/10.1016/j.aquaculture.2011.06.032

SUI J, Luan S, Luo K, Meng X, Lu X, Cao B, Li W, Chai Z, Liu N, Xu S, Kong J. 2016. Genetic parameters and response to selection for harvest body weight of Pacific white shrimp, Litopenaeus vannamei. Aquaculture Research. 47(9):2795-2803. ISSN: 1355557X. https://doi.org/10.1111/are.12729

TAN J, Luan S, Luo K, Guan J, Li W, Sui J, Guo Z, Xu S, Kong J. 2017. Heritability and genotype by environment interactions for growth and survival in Litopenaeus vannamei at low and high densities. Aquaculture Research. 48(4):1430-1438. ISSN: 1355-557X. https://doi.org/10.1111/are.12978

THOA NP, Knibb W, Ninh NH, Van Dai N, Nhat PH, Nguyen NH. 2015. Genetic variation in survival of tilapia (Oreochromis niloticus, Linnaeus, 1758) fry during the early phase of rearing in brackish water environment (5-10 ppt). Aquaculture. 442:112-118. ISSN: 00448486. https://doi.org/10.1016/j.aquaculture.2015.02.040

TRANG TT, Hung NH, Ninh NH, Knibb W, Nguyen NH. 2019. Genetic Variation in Disease Resistance Against White Spot Syndrome Virus (WSSV) in Liptopenaeus vannamei. Frontiers in Genetics. 10:264. ISSN: 1664-8021. https://doi.org/10.3389/fgene.2019.00264

VEHVILÄINEN H, Kause A, Koskinen H, Paananen T. 2010. Genetic architecture of rainbow trout survival from egg to adult. Genetics research. 92(1):1-11. ISSN: 0016-6723. https://doi.org/10.1017/S0016672310000017

VEHVILÄINEN H, Kause A, Quinton C, Koskinen H, Paananen T. 2008. Survival of the currently fittest: genetics of rainbow trout survival across time and space. Genetics. 180(1):507-516. ISSN: 0016-6731. https://doi.org/10.1534/genetics.108.089896

YUAN R, Hu Z, Liu J, Zhang J. 2018. Genetic parameters for growth-related traits and survival in Pacific white shrimp, Litopenaeus vannamei under conditions of high ammoniaN concentrations. Turkish Journal of Fisheries and Aquatic Sciences. 18(1):37-47. ISSN: 1303-2712. https://doi.org/10.4194/1303-2712-v18_1_05

Zhang J, Cao F, Liu J, Yuan R. 2017. Genetic parameters for growth and survival traits in Litopenaeus vannamei at different ages. Aquaculture International. 25(5):1901-1911. ISSN: 0967-6120. https://doi.org/10.1007/s10499-017-0163-9 